\title{
Confluence up to Garbage
}

\author{
Graham Campbell ${ }^{1(\bowtie)}\left(\mathbb{C}\right.$ and Detlef Plump ${ }^{2}$ (1) \\ 1 School of Mathematics, Statistics and Physics, Newcastle University, \\ Newcastle upon Tyne, UK \\ $\mathrm{g} \cdot \mathrm{j}$. campbell2@newcastle.ac.uk \\ 2 Department of Computer Science, University of York, York, UK \\ detlef.plump@york.ac.uk
}

\begin{abstract}
The transformation of graphs and graph-like structures is ubiquitous in computer science. When a system is described by graphtransformation rules, it is often desirable that the rules are both terminating and confluent so that rule applications in an arbitrary order produce unique resulting graphs. However, there are application scenarios where the rules are not globally confluent but confluent on a subclass of graphs that are of interest. In other words, non-resolvable conflicts can only occur on graphs that are considered as "garbage". In this paper, we introduce the notion of confluence up to garbage and generalise Plump's critical pair lemma for double-pushout graph transformation, providing a sufficient condition for confluence up to garbage by non-garbage critical pair analysis. We apply our results to language recognition by backtracking-free graph reduction, showing how to establish that a graph language can be decided by a system which is confluent up to garbage. We present two case studies with backtracking-free graph reduction systems which recognise a class of flow diagrams and a class of labelled series-parallel graphs, respectively. Both systems are non-confluent but confluent up to garbage.
\end{abstract}

Keywords: Graph transformation - Confluence • Graph languages • Decision procedures

\section{Introduction}

Rule-based graph transformation and graph grammars date back to the late 1960s. The best developed theoretical framework is the so-called double-pushout (DPO) approach to graph transformation [10,12]. When specifying systems in computer science by DPO graph transformation rules, it is often desirable that the rules are both terminating and confluent so that rule applications in an arbitrary order produce unique resulting graphs. However, there are application scenarios where the rules are not confluent but confluent on a subclass of graphs

G. Campbell-Supported by a Vacation Internship and a Doctoral Training Grant No. (2281162) from the Engineering and Physical Sciences Research Council (EPSRC) in the UK, while at University of York and Newcastle University, respectively.

(C) Springer Nature Switzerland AG 2020

F. Gadducci and T. Kehrer (Eds.): ICGT 2020, LNCS 12150, pp. 20-37, 2020.

https://doi.org/10.1007/978-3-030-51372-6_2 
that are of interest. In other words, non-resolvable conflicts can only occur on graphs that are considered as "garbage".

In this paper, we introduce the notions of (local) confluence up to garbage and termination up to garbage in graph transformation. We generalise Plump's Critical Pair Lemma [26,28] and Newmann's Lemma [25] and thereby allow to check confluence up to garbage via non-garbage critical pair analysis. We apply our results to language recognition by backtracking-free graph reduction, showing how to establish that a graph language can be decided by a system which is confluent up to garbage. We present two case studies with backtracking-free graph reduction systems which recognise a class of flow diagrams and a class of labelled series-parallel graphs, respectively. Both systems are non-confluent but confluent up to garbage. Parts of this paper are based on Chapter 4 of an unpublished report [6], in turn developed from Campbell's BSc Thesis [5].

\section{Preliminaries}

We review some terminology for binary relations, the DPO approach to graph transformation, graph languages, and confluence checking.

\subsection{Abstract Reduction Systems}

An abstract reduction system $(\mathrm{ARS})$ is a pair $(A, \rightarrow)$ where $A$ is a set and $\rightarrow$ a binary relation on $A$. We say that:

1. $y$ is a successor to $x$ if $x \stackrel{+}{\longrightarrow} y$, and a direct successor if $x \rightarrow y$;

2. $x$ and $y$ are joinable if there is a $z$ such that $x \stackrel{*}{\rightarrow} z \stackrel{*}{\leftarrow} y$. We write $x \downarrow y$;

3. $\rightarrow$ is confluent if $y_{1} \stackrel{*}{\leftarrow} x \stackrel{*}{\rightarrow} y_{2}$ implies $y_{1} \downarrow y_{2}$;

4. $\rightarrow$ is locally confluent if $y_{1} \leftarrow x \rightarrow y_{2}$ implies $y_{1} \downarrow y_{2}$;

5 . $\rightarrow$ is terminating if there is no infinite sequence $x_{0} \rightarrow x_{1} \rightarrow \ldots$

The principle of Noetherian induction is:

$$
\frac{\forall x \in A,(\forall y \in A, x \stackrel{+}{\longrightarrow} y \Rightarrow P(y)) \Rightarrow P(x)}{\forall x \in A, P(x)}
$$

Theorem 1 (Noetherian Induction [1]). Given an $A R S(A, \rightarrow)$, the principle of Noetherian induction holds if and only if $\rightarrow$ is terminating.

Theorem 2 (Newman's Lemma [25]). A terminating relation is confluent if and only if it is locally confluent.

\subsection{Labelled Graphs and Morphisms}

We will be working with directed labelled graphs [15]. An alphabet is a pair $\Sigma=\left(\Sigma_{V}, \Sigma_{E}\right)$ of finite sets of node and edge labels from which a graph can be labelled. A graph (over $\Sigma$ ) is a tuple $G=(V, E, s, t, l, m)$ where $V$ is a finite set 
of nodes, $E$ is a finite set of edges, $s: E \rightarrow V$ is the source function, $t: E \rightarrow V$ is the target function, $l: V \rightarrow \Sigma_{V}$ is the node labelling function, and $m: E \rightarrow \Sigma_{E}$ is the edge labelling function. We may write the components as $V_{G}, E_{G}, s_{G}$, etc.

A graph morphism $g: G \rightarrow H$ is a pair $g=\left(g_{V}, g_{E}\right)$ of functions $g_{V}: V_{G} \rightarrow$ $V_{H}$ and $g_{E}: E_{G} \rightarrow E_{H}$ such that $g_{V} \circ s_{G}=s_{H} \circ g_{E}, g_{V} \circ t_{G}=t_{H} \circ g_{E}, l_{G}=l_{H} \circ g_{V}$ and $m_{G}=m_{H} \circ g_{E}$. We say $g$ is injective (surjective, bijective) if both functions $g_{V}$ and $g_{E}$ are. A graph $H$ is a subgraph of $G$, denoted by $H \subseteq G$, if there exists an inclusion morphism $i: H \rightarrow G$ with $i(x)=x$ for all items $x$.

It is well known that graphs and morphisms over $\Sigma$ form a category. Graph morphisms are bijective if and only if they are isomorphisms in the categorical sense. Given a graph $G$, we write $[G]$ for the isomorphism class of $G$ and call $[G]$ an abstract graph. We denote by $\mathcal{G}(\Sigma)$ the set of all abstract graphs over $\Sigma$.

\subsection{Double-Pushout Graph Transformation}

A rule is a pair of inclusions $r=\langle L \leftarrow K \rightarrow R\rangle$, where $L$ is the left-hand side (LHS), $K$ the interface, and $R$ the right-hand side (RHS). A match of $r$ in a graph $G$ is an injective morphism $L \rightarrow G$. An application of rule $\mathrm{r}$ to $G$ with match $g: L \rightarrow G$ requires to construct two pushouts as in Fig. 1. We write $G \Rightarrow_{r, g} H$ for this application and call the diagram in Fig. 1 a direct derivation.

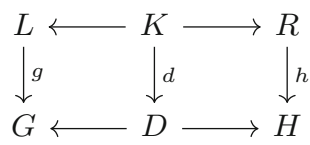

Fig. 1. A direct derivation

Given $r$ and the match $g: L \rightarrow G$, the direct derivation of Fig. 1 exists if and only if the dangling condition is satisfied: nodes in $g(L-K)$ must not be incident to edges in $G-g(L)$. In this case the graphs $D$ and $H$ are determined uniquely up to isomorphism [10]. We call the injective morphism $h$ the comatch of the rule application.

Given a set of rules $\mathcal{R}$, we write $G \Rightarrow_{\mathcal{R}} H$ if $H$ is obtained from $G$ by applying any of the rules from $\mathcal{R}$. We write $G \Rightarrow_{\mathcal{R}}^{+} H$ if $H$ is obtained from $G$ by one or more rule applications, and $G \Rightarrow_{\mathcal{R}}^{*} H$ if $G \cong H$ or $G \Rightarrow_{\mathcal{R}}^{+} H$.

By pushout properties, the relation $\Rightarrow_{\mathcal{R}}$ can be lifted to abstract graphs. Hence we have an ARS $\left(\mathcal{G}(\Sigma), \Rightarrow_{\mathcal{R}}\right)$. This view gives us the definition of (local) confluence and termination for graph transformation systems.

\subsection{Graph Languages}

A graph language is simply a set of graphs, in the same way that a string language is a set of strings. Just like we can define string languages using string grammars, 
we can define graph languages using graph grammars, where we rewrite some start graph using a set of graph transformation rules. Derived graphs are then defined to be in the language exactly when they are terminally labelled.

Given a graph transformation system $T=(\Sigma, \mathcal{R})$, a subalphabet of nonterminals $\mathcal{N}$, and a start graph $S$ over $\Sigma$, then a graph grammar is a tuple $\mathcal{G}=(\Sigma, \mathcal{N}, \mathcal{R}, S)$. We say that a graph $G$ is terminally labelled if $l(V) \cap \mathcal{N}_{V}=\emptyset$ and $m(E) \cap \mathcal{N}_{E}=\emptyset$. Thus, we can define the graph language generated by $\mathcal{G}$ :

$$
\mathrm{L}(\mathcal{G})=\left\{[G] \mid S \Rightarrow_{\mathcal{R}}^{*} G, G \text { terminally labelled }\right\} .
$$

Given $\mathcal{G}=(\Sigma, \mathcal{N}, \mathcal{R}, S)$, we have $G \Rightarrow_{r} H$ if and only if $H \Rightarrow_{r^{-1}} G$, for some $r \in \mathcal{R}$, by using the comatch. Moreover, $[G] \in \mathrm{L}(\mathcal{G})$ if and only if $G \Rightarrow_{\mathcal{R}^{-1}}^{*} S$ and $G$ is terminally labelled. So we have a non-deterministic membership checking.

\subsection{Confluence Checking}

In 1970, Knuth and Bendix showed that confluence checking of terminating term rewriting systems is decidable [18]. Moreover, it suffices to compute all critical pairs and check their joinability $[1,17]$. Unfortunately, for (terminating) graph transformation systems, confluence is not decidable in general, and joinability of critical pairs does not imply local confluence. In 1993, Plump showed that strong joinability of all critical pairs is sufficient but not necessary to show local confluence [26,28].

The derivations $H_{1} \Leftarrow_{r_{1}, g_{1}} G \Rightarrow_{r_{2}, g_{2}} H_{2}$ are parallelly independent if $\left(g_{1}\left(L_{1}\right) \cap\right.$ $\left.g_{2}\left(L_{2}\right)\right) \subseteq\left(g_{1}\left(K_{1}\right) \cap g_{2}\left(K_{2}\right)\right)$. We say two parallelly independent derivations are a critical pair if additionally $G=g_{1}\left(L_{1}\right) \cup g_{2}\left(L_{2}\right)$, and if $r_{1}=r_{2}$ then $g_{1} \neq g_{2}$. Every graph transformation system has only finitely many critical pairs.

Let $G \Rightarrow H$ be a direct derivation. Then the track morphism is defined to be the partial morphism $\operatorname{tr}_{G \Rightarrow H}=i n^{\prime} \circ i n^{-1}$, where $i n$ and $i n^{\prime}$ are the bottom left and right morphisms in Fig. 1, respectively. We define $\operatorname{tr}_{G \Rightarrow^{*} H}$ inductively as the composition of track morphisms. The set of persistent nodes of a critical pair $\Phi: H_{1} \Leftarrow G \Rightarrow H_{2}$ is Persist $_{\Phi}=\left\{v \in G_{V} \mid \operatorname{tr}_{G \Rightarrow H_{1}}(\{v\}), \operatorname{tr}_{G \Rightarrow H_{2}}(\{v\}) \neq \emptyset\right\}$. That is, those nodes that are not deleted by the application of either rule.

A critical pair $\Phi: H_{1} \Leftarrow G \Rightarrow H_{2}$ is strongly joinable if it is joinable without deleting any of the persistent nodes, and the persistent nodes are identified when joining. That is, there exists a graph $M$ and derivations $H_{1} \Rightarrow_{\mathcal{R}}^{*} M \Leftarrow_{\mathcal{R}}^{*} H_{2}$ such that $\forall v \in$ Persist $_{\Phi}, \operatorname{tr}_{G \Rightarrow H_{1} \Rightarrow * M}(\{v\})=\operatorname{tr}_{G \Rightarrow H_{2} \Rightarrow^{*} M}(\{v\}) \neq \emptyset$.

Theorem 3 (Critical Pair Lemma [26,28]). A graph transformation system $T$ is locally confluent if all its critical pairs are strongly joinable.

The original proof of the Critical Pair Lemma needs the Commutativity, Clipping and Embedding Theorems, and some auxiliary definitions. We will need these intermediate results when we come to prove our generalised version.

Theorem 4 (Commutativity [11]). If $H_{1} \Leftarrow_{r_{1}, g_{1}} G \Rightarrow_{r_{2}, g_{2}} H_{2}$ are parallelly independent, then there is a graph $G^{\prime}$ and derivations $H_{1} \Rightarrow_{r_{2}} G^{\prime} \Leftarrow_{r_{1}} H_{2}$. 
Let the derivation $\Delta: G_{0} \Rightarrow^{*} G_{n}$ be given by pushouts $(1),\left(1^{\prime}\right), \ldots,(n),\left(n^{\prime}\right)$ and suppose there are pushouts $(\underline{1}),\left(\underline{1^{\prime}}\right), \ldots,(\underline{n}),\left(\underline{n^{\prime}}\right)$ whose vertical morphisms are injective (Fig. 2). Then, the derivation $\Delta^{\prime}: G_{0}^{\prime} \Rightarrow^{*} G_{n}^{\prime}$ consisting of the composed pushouts $(1+\underline{1}), \ldots,\left(n^{\prime}+\underline{n^{\prime}}\right)$ is an instance of $\Delta$ based on the morphism $G_{0} \rightarrow G_{0}^{\prime}$. Moreover, we define the subgraph Use $\Delta$ to be all items $x$ such that there is some $i \geq 0$ with $G_{0} \Rightarrow^{*} G_{i}(x) \in \operatorname{Match}\left(G_{i} \Rightarrow G_{i+1}\right)$ where $\operatorname{Match}\left(G_{i} \Rightarrow G_{i+1}\right)$ is the image of the associated rule's left hand side graph under the match $L \rightarrow G_{i}$.

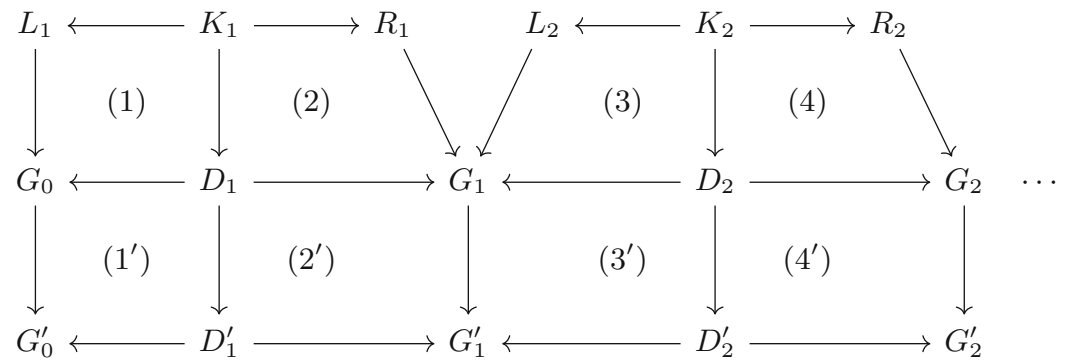

Fig. 2. Derivation instances

Theorem 5 (Clipping [27]). Given a derivation $\Delta^{\prime}: G^{\prime} \Rightarrow^{*} H^{\prime}$ and an injective morphism $h: G \rightarrow G^{\prime}$ such that Use $\Delta^{\prime} \subseteq h(G)$, there exists a derivation $\Delta: G \Rightarrow^{*} H$ such that $\Delta^{\prime}$ is an instance of $\Delta$ based on $h$.

Given a derivation $\Delta: G \Rightarrow^{*} H$ the subgraph of $G$, Persist $\Delta$, consists of all items $x$ such that $\operatorname{tr}_{G \Rightarrow^{*} H}(x)$ is defined.

Theorem 6 (Embedding [27]). Let $\Delta: G \Rightarrow^{*} H$ be a derivation, $h: G \rightarrow G^{\prime}$ an injective graph morphism, $B_{\Delta}$ be the discrete subgraph of $G$ consisting of all nodes $x$ such that $h(x)$ is incident to an edge in $G^{\prime} \backslash h(G)$. If $B_{\Delta} \subseteq$ Persist $\Delta$, then there exists a derivation $\Delta^{\prime}: G^{\prime} \Rightarrow^{*} H^{\prime}$ such that $\Delta^{\prime}$ is an instance of $\Delta$ based on $h$. Moreover, there exists a pushout of $t: B_{\Delta} \rightarrow H$ along $h^{\prime}: B_{\Delta} \rightarrow C_{\Delta}$ where $C_{\Delta}=\left(G^{\prime} \backslash h(G)\right) \cup h\left(B_{\Delta}\right)$ and $t$ is the restriction of $\operatorname{tr}_{G \Rightarrow^{*} H}$ to $B_{\Delta}$.

\section{Closedness and Confluence up to Garbage}

In this section, we introduce (local) confluence and termination up to garbage, and closedness. We show that if we have closedness and termination up to garbage, then local confluence up to garbage implies confluence up to garbage: the Generalised Newmann's lemma. Moreover, we recap that closedness is undecidable in general, in the context of DPO graph transformation. 


\subsection{Closedness and Garbage}

Definition 1. Let $T=(\Sigma, \mathcal{R})$ be a GT system, and $\mathcal{D} \subseteq \mathcal{G}(\Sigma)$ be a set of abstract graphs. Then, a graph $G$ is called garbage if $[G] \notin \mathcal{D}$ and $\mathcal{D}$ is closed under $T$ if for all $G, H$ such that $G \Rightarrow_{\mathcal{R}} H$, if $[G] \in \mathcal{D}$ then $[H] \in \mathcal{D}$.

The idea is that a set of abstract graphs $\mathcal{D}$ represents the good input, and the garbage is the graphs that are not in this set. $\mathcal{D}$ need not be explicitly generated by a graph grammar. For example, it could be defined by some (monadic secondorder [8]) logical formula.

Example 1. Consider the reduction rules in Fig. 3. The language of acyclic graphs is closed under the GT system $\left((\{\square\},\{\square\}),\left\{r_{1}\right\}\right)$, and the language of trees (forests) and its complement are both closed under $\left((\{\square\},\{\square\}),\left\{r_{2}\right\}\right)$.

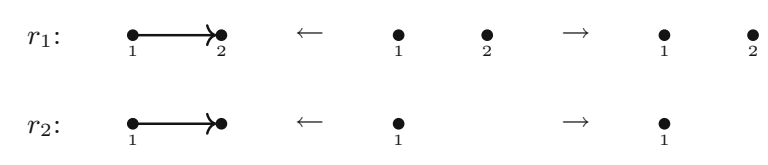

Fig. 3. Example reduction rules

\section{Definition 2 (Closedness Problem).}

Input: A GT system $T=(\Sigma, \mathcal{R})$ and a graph grammar $\mathcal{G}$ over $\Sigma$.

Question: Is $\mathrm{L}(\mathcal{G})$ closed under $T$ ?

It turns out that closedness is undecidable in general, even if we restrict ourselves to recursive languages and terminating GT systems. In 1998, Fradet and Le Métayer showed the following result:

Theorem 7 (Undecidable Closedness [14]). The closedness problem is undecidable in general, even for terminating GT systems $T$ with only one rule, and $\mathcal{G}$ an edge replacement grammar.

\subsection{Confluence up to Garbage}

We can now define (local) confluence and termination up to garbage, allowing us to say that, ignoring the garbage graphs, a system is (locally) confluent.

Definition 3. Let $T=(\Sigma, \mathcal{R}), \mathcal{D} \subseteq \mathcal{G}(\Sigma)$. Then:

1. if for all graphs $G, H_{1}, H_{2}$, such that $[G] \in \mathcal{D}, H_{1} \Leftarrow_{\mathcal{R}} G \Rightarrow_{\mathcal{R}} H_{2}$ implies that $H_{1}, H_{2}$ are joinable, then $T$ is locally confluent (up to garbage) on $\mathcal{D}$;

2. if for all graphs $G, H_{1}, H_{2}$, such that $[G] \in \mathcal{D}, H_{1} \Leftarrow{ }_{\mathcal{R}}^{*} G \Rightarrow_{\mathcal{R}}^{*} H_{2}$ implies that $H_{1}, H_{2}$ are joinable, then $T$ is confluent (up to garbage) on $\mathcal{D}$; 
3. if there is no infinite derivation sequence $G_{0} \Rightarrow_{\mathcal{R}} G_{1} \Rightarrow_{\mathcal{R}} G_{2} \Rightarrow_{\mathcal{R}} \cdots$ such that $\left[G_{0}\right] \in \mathcal{D}$, then $T$ is terminating (up to garbage) on $\mathcal{D}$.

The following is an immediate consequence of set inclusion:

Proposition 1. Let $T=(\Sigma, \mathcal{R}), \mathcal{D} \subseteq \mathcal{G}(\Sigma), \mathcal{E} \subseteq \mathcal{D}$. Then (local) confluence on $\mathcal{D}$ implies (local) confluence on $\mathcal{E}$, and similarly for termination.

Example 2. Looking again at $r_{1}$ and $r_{2}$ from our first example, it is easy to see that $r_{1}$ is terminating and confluent up to garbage on the language of acyclic graphs, but is not confluent on all graphs. Similarly, $r_{2}$ is terminating and confluent up to garbage on the language of trees.

Example 3. Consider the rules in Fig. 4. Clearly they are terminating, since they are size reducing. Moreover, the language of all linked lists with edge labels $a$ or $b$ and its complement are closed under the rules. The rules are not locally confluent. To see this, consider the 3 -cycle with edges labelled with $a, a, b$. It is possible for the cycle to be reduced to either the 2-cycle with edges $a$ and $a$ or the 2-cycle with edge $a$ and $b$. Neither of these cycles can be reduced further, and so we have a counter example to confluence. These rules are locally confluent on linked lists. Moreover, an input graph $G$ is a linked list if and only if it can be reduced using these rules to a length one linked list.

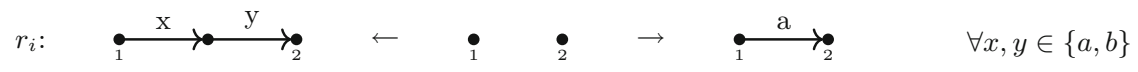

Fig. 4. List reduction rules

Theorem 8 (Generalised Newman's Lemma). Let $T=(\Sigma, \mathcal{R}), \mathcal{D} \subseteq$ $\mathcal{G}(\Sigma)$. If $T$ is terminating on $\mathcal{D}$ and $\mathcal{D}$ is closed under $T$, then $T$ is confluent on $\mathcal{D}$ if and only if it is locally confluent on $\mathcal{D}$.

Proof. This can be seen by Noetherian Induction (Fig. 5), due to the fact that closedness ensures applicability of the induction hypothesis. 


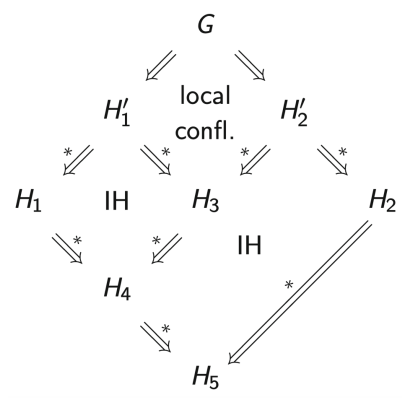

Fig. 5. Induction step diagram

\section{Generalised Critical Pair Lemma}

In this section, we generalise Plump's Critical Pair Lemma, providing a machine checkable sufficient condition for local confluence up to garbage. For this, we need to define a notion of subgraph closure and non-garbage critical pairs.

\subsection{Subgraph Closure}

In the proof of the traditional critical pair lemma for (hyper)graphs, the argument is that if a pair of derivations is not parallelly independent, then it must be the case that a critical pair can be embedded within it. In our new setting, the possible start graphs will be restricted, since some of the graphs will be garbage. We are only interested in those critical pairs with start graphs that can be embedded in non-garbage graphs. This is exactly the statement that the start graph of the critical pair is in the subgraph closure of the non-garbage graphs.

Definition 4. Let $\mathcal{D} \subseteq \mathcal{G}(\Sigma)$ be a set of abstract graphs. Then $\mathcal{D}$ is subgraph closed if for all graphs $G, H$, such that $H \subseteq G$, if $[G] \in \mathcal{D}$, then $[H] \in \mathcal{D}$. The subgraph closure of $\mathcal{D}$, denoted $\widehat{\mathcal{D}}$, is the smallest set containing $\mathcal{D}$ that is subgraph closed.

Proposition 2. Given $\mathcal{D} \subseteq \mathcal{G}(\Sigma), \widehat{\mathcal{D}}$ always exists, and is unique. Moreover, $\mathcal{D}=\widehat{\mathcal{D}}$ if and only if $\mathcal{D}$ is subgraph closed.

Proof. The key observations are that the subgraph relation is transitive, and each graph has only finitely many subgraphs. Clearly, the smallest possible set containing $\mathcal{D}$ is just the union of all subgraphs of the elements of $\mathcal{D}$, up to isomorphism. This is the unique subgraph closure of $\mathcal{D}$.

$\widehat{\mathcal{D}}$ always exists, however it need not be decidable, even when $\mathcal{D}$ is! It is not obvious what conditions on $\mathcal{D}$ ensure that $\widehat{\mathcal{D}}$ is decidable. Interestingly, the classes of regular and context-free string languages are actually closed under substring closure [4]. 
Example 4. $\emptyset, \mathcal{G}(\Sigma)$, and the language of discrete graphs are subgraph closed.

Example 5. The subgraph closure of the language of trees is the language of forests. The subgraph closure of the language of connected graphs is the language of all graphs.

\subsection{Non-garbage Critical Pairs}

We now define non-garbage critical pairs, which allow us to ignore certain pairs, which if all are strongly joinable, will allow us to conclude local confluence up to garbage, even in the presence of (local) non-confluence on all graphs.

Definition 5. Let $T=(\Sigma, \mathcal{R}), \mathcal{D} \subseteq \mathcal{G}(\Sigma)$. A critical pair $H_{1} \Leftarrow G \Rightarrow H_{2}$ is non-garbage if $[G] \in \widehat{\mathcal{D}}$.

Lemma 1. Given a $G T$ system $T=(\Sigma, \mathcal{R})$ and $\mathcal{D} \subseteq \mathcal{G}(\Sigma)$, then there are only finitely many non-garbage critical pairs up to isomorphism. Moreover, if $\widehat{\mathcal{D}}$ is decidable, then one can find them in finite time.

Proof. There are only finitely many critical pairs for $T$, up to isomorphism, and there exists a terminating procedure for generating them. It then remains to filter out the garbage pairs, which can always be done if $\widehat{\mathcal{D}}$ is decidable.

Corollary 1. Let $T=(\Sigma, \mathcal{R}), \mathcal{D} \subseteq \mathcal{G}(\Sigma)$ be such that $T$ is terminating on $\mathcal{D}$ and $\widehat{\mathcal{D}}$ is decidable. Then, one can decide if all the non-garbage critical pairs are strongly joinable.

Proof. By Lemma 1, we can generate all the pairs, but then since $T$ is terminating on $D$, there are only finitely many successor graphs to be generated. We can then test each for strong joinability in finite time.

Theorem 9 (Generalised Critical Pair Lemma). Let $T=(\Sigma, \mathcal{R}), \mathcal{D} \subseteq$ $\mathcal{G}(\Sigma)$. If all its non-garbage critical pairs are strongly joinable, then $T$ is locally confluent on $\mathcal{D}$.

Proof. Our proof is a generalisation of Plump's original proof of the Critical Pair Lemma for (hyper)graphs (Theorem 3) [26,28]. We need to show that every pair of derivations $H_{1} \Leftarrow{ }_{r_{1}, g_{1}} G \Rightarrow_{r_{2}, g_{2}} H_{2}$ such that $G$ is non-garbage can be joined. There are two cases to consider. Firstly, if the derivations are parallelly independent, then by Theorem 4, the result is immediate. Otherwise, we must consider the case that they are not parallelly independent.

By Theorem 5 , we can factor out a pair $T_{1} \Leftarrow S \Rightarrow T_{2}$. Since critical pairs are, by construction, the overlaps of rule left hand sides, it must be the case that this pair is actually a critical pair. Moreover, since $[G] \in \mathcal{D}$, then $[S] \in \widehat{\mathcal{D}}$ and so the critical pair must be non-garbage, and must be strongly joinable to $U$. We can now apply Theorem 6 to $T_{1} \Rightarrow^{*} U$ and $T_{2} \Rightarrow^{*} U$, separately, giving result graphs $M_{1}$ and $M_{2}$ (applicability of the theorem is a consequence of strong joinability). To see that $M_{1}$ and $M_{2}$ are isomorphic follows from elementary properties of pushouts along monomorphisms [28] (Fig.6). 


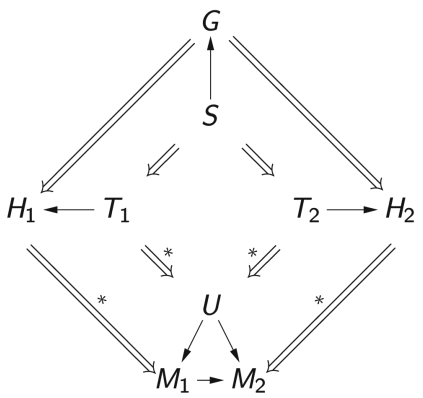

Fig. 6. Generalised critical pair lemma diagram

Corollary 2. Let $T=(\Sigma, \mathcal{R}), \mathcal{D} \subseteq \mathcal{G}(\Sigma)$. If $T$ is terminating on $\mathcal{D}, \mathcal{D}$ is closed under $T$, and all $T$ 's non-garbage critical pairs are strongly joinable, then $T$ is confluent on $\mathcal{D}$.

Proof. By the above theorem, $T$ is locally confluent up to garbage, so by the Generalised Newman's Lemma (Theorem 8), $T$ is confluent up to garbage.

Obviously, checking for local confluence up to garbage is undecidable in general, even when $\widehat{\mathcal{D}}$ is decidable and the system is terminating and closed. What is remarkable though, is that local confluence up to garbage is actually undecidable in general for a terminating non-length-increasing string rewriting systems and $\mathcal{D}$ a regular string language [7]!

\subsection{Checking for Confluence up to Garbage}

Given a GT system $T$ and a language $\mathcal{D}$ (possibly specified by a grammar), the process is to:

1. Establish (by means of direct proof) that $T$ is terminating on $\mathcal{D}$ and $\mathcal{D}$ is closed under $T$. If this is not true, one may want to restart with some language containing $\mathcal{D}$ to try to establish closedness.

2. Generate the finitely many non-garbage critical pairs of $T$.

3. Check if each generated pair is (strongly) joinable.

If all the pairs are strongly joinable, then we have confluence up to garbage due to Corollary 2. If all the pairs are joinable, but not all strongly, then we cannot draw any conclusions, but one may be able to construct a counter example to confluence by attaching context to nodes. Finally, if one of the pairs is not joinable at all, then we have a direct counter example to confluence, and we can conclude non-confluence up to garbage.

\section{Language Recognition}

In this section, we introduce a general notion of what it means to recognise a language, and what it means to be a confluent decider. We then demonstrate the 
applicability of our earlier results by showing that there are confluent deciders for Extended Flow Diagrams and Labelled Series-Parallel Graphs, even in the absence of confluence. We thus have algorithms, specified by reduction rules, that can check membership of these languages without needing to backtrack.

\subsection{Confluent Recognition}

One can think of graph transformation systems in terms of grammars that define languages. If they are terminating, then membership testing is decidable, but in general, non-deterministic in the sense that a deterministic algorithm must backtrack if it produces a normal form not equal to the start graph, to determine if another derivation sequence could have reached it. If the system is confluent too, then the algorithm becomes deterministic.

In general, the requirement of confluence is too strong, and one only requires confluence on the language we are recognising. Using the results from the last section, it is often possible to prove local confluence up to garbage using the Generalised Critical Pair Lemma, and then, in the presence of termination and closure, use the Generalised Newman's Lemma to show confluence up to garbage. Closedness and language recognition has actually been considered before by Bakewell, Plump, and Runciman, in the context of languages specified by reduction systems without non-terminals [3], but without the development of the theory we have provided.

Before continuing, we must provide a formal definition of what it means to recognise a language, and that grammars satisfy our definition by considering their rules in reverse, abstracting away from grammars, with a more general definition that accounts for the fact that reduction systems may need auxiliary symbols, not in the input, in the same way grammars can use non-terminals.

Definition 6 (Language Recognition). Let $T=(\Sigma, \mathcal{R})$ be a GT system, $\mathcal{I} \subseteq \Sigma$ an input alphabet, and $\mathcal{S}$ a finite set of graphs over $\Sigma$. Then we say that $(T, \mathcal{S})$ recognises a language $\mathcal{L}$ over $\mathcal{I}$ if for all graphs $G$ over $\mathcal{I},[G] \in \mathcal{L}$ if and only if $G \Rightarrow_{\mathcal{R}}^{*} S$ for some $S \in \mathcal{S}$.

Theorem 10 (Membership Checking). Given a grammar $\mathcal{G}=(\Sigma, \mathcal{N}, \mathcal{R}$, $S),[G] \in \mathrm{L}(\mathcal{G})$ if and only if $G \Rightarrow_{\mathcal{R}^{-1}}^{*} S$ and $G$ is terminally labelled. That is, $\left(\left(\Sigma, \mathcal{R}^{-1}\right),\{S\}\right)$ recognises $\mathrm{L}(\mathcal{G})$ over $\Sigma \backslash \mathcal{N}$.

Proof. The key is that rules and derivations are invertible, which means that if $S$ can be derived from $G$ using the reverse rules, then $G$ can be derived from $S$ using the original rules so is in the language. If $S$ cannot be derived from $G$, then $G$ cannot be in the language since that would imply there was a derivation sequence from $S$ to $G$ which we could invert to give a contradiction.

We are now ready to define confluent deciders, and show that such systems can test for language membership without backtracking. 
Definition 7 (Confluent Decider). Let $T=(\Sigma, \mathcal{R})$ be a GT system, $\mathcal{I} \subseteq \Sigma$ an input alphabet, and $\mathcal{S}$ a finite set of graphs over $\Sigma$. Then we say that $(T, \mathcal{S})$ is a confluent decider for a language $\mathcal{L}$ over $\mathcal{I}$ if $(T, \mathcal{S})$ recognises $\mathcal{L}$ over $\mathcal{I}, T$ is terminating on $\mathcal{G}(\mathcal{I})$, and $T$ is confluent on $\mathcal{L}$.

Theorem 11 (Confluent Decider Correctness). Given a confluent decider $(T, \mathcal{S})$ for a language $\mathcal{L}$ over $\mathcal{I} \subseteq \Sigma$ and an input graph $G$ over $\mathcal{I}$, the following algorithm is correct: Compute a normal form of $G$ by deriving successor graphs using $T$ as long as possible. If the result graph is isomorphic to $S$, the input graph is in the language. Otherwise, the graph is not in the language.

Proof. Suppose $G$ is not in $\mathcal{L}$. Then, since $T$ is terminating on $\mathcal{G}(\mathcal{I})$ our algorithm must be able to find a normal form of $G$, say $H$, and because $T$ recognises $\mathcal{L}$, it must be the case that $H$ is not isomorphic to $S$, and so the algorithm correctly decides that $G$ is not in $\mathcal{L}$.

Now, suppose that $G$ is in $\mathcal{L}$. Then, because $T$ is terminating, as before, we must be able to derive some normal form, $H$. But then, since $T$ is both confluent on $\mathcal{L}$ and recognises $\mathcal{L}$, it must be the case that $H$ is isomorphic to $S$, and so the algorithm correctly decides that $G$ is in $\mathcal{L}$.

What we really want is a version of Theorem 10 for instantiating confluent deciders. We really want is a Since both termination and confluence testing is undecidable in general, we cannot hope for an effective procedure, even for a terminating system, however the theory we introduced in the previous sections will help by automating local confluence checking. It just remains for us to choose a suitable set $\mathcal{D}$, and proceed in a similar way to as described in Subsect. 4.3. For the remainder of this section, we will look at two examples that demonstrate how we can use the Generalised Newman's Lemma and Generalised Critical Pair Lemma to show that we have a confluent decider for a language, given a grammar that generates the language.

\subsection{Extended Flow Diagrams}

In 1976, Farrow, Kennedy and Zucconi presented semi-structured flow graphs, defining a grammar with confluent reduction rules [13]. Plump has considered a restricted version of this language: extended flow diagrams (EFDs) [28]. The reduction rules for extended flow diagrams are a confluent decider for the EFDs, despite not being confluent.

Definition 8. The language of extended flow diagrams is generated by EFD = $(\Sigma, \mathcal{N}, \mathcal{R}, S)$ where $\Sigma_{V}=\{\bullet, \square, \diamond\}, \Sigma_{E}=\{t, f, \square\}, \mathcal{N}_{V}=\mathcal{N}_{E}=\emptyset, \mathcal{R}=$ $\{$ seq, while, ddec, dec $1, \operatorname{dec} 2\}$, and $S=\bullet \square \rightarrow \bullet$ (Fig. 7).

In the next figure, the shorthand notation with the numbers under the nodes places such nodes in the interface graph of the rules. We assume that the interface graphs are discrete (have no edges). 


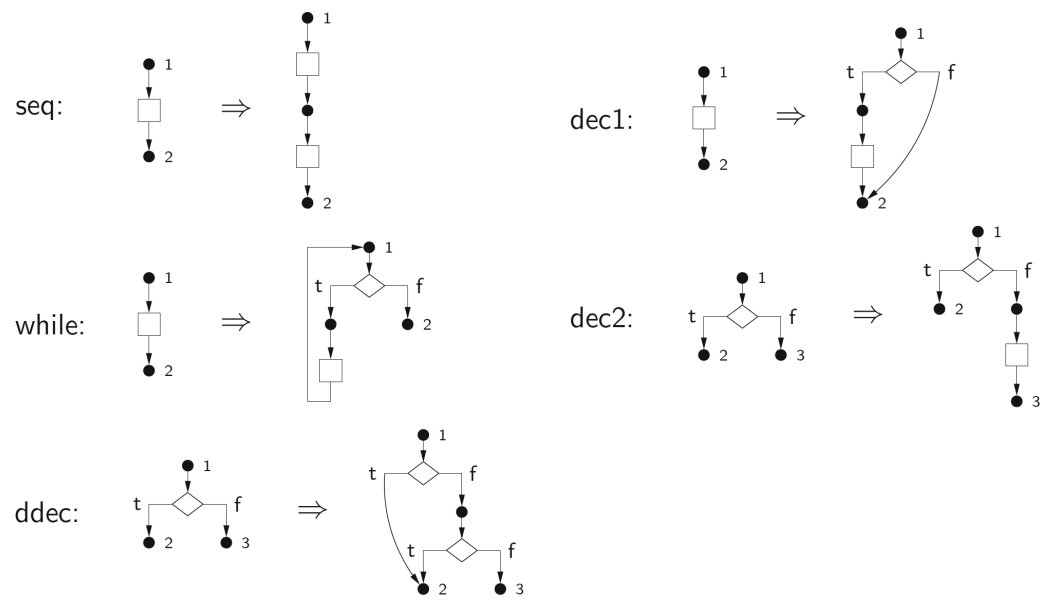

Fig. 7. EFD grammar rules

Lemma 2. Every directed cycle in an EFD contains a t-labelled edge

Proof. By induction.

Theorem 12 (Confluent EFD Decider). Let $T=\left(\Sigma, \mathcal{R}^{-1}\right)$. Then $(T,\{S\})$ is a confluent decider for $\mathrm{L}(\mathrm{EFD})$ over $\Sigma$.

Proof. By Theorem 10, $T$ recognises $\mathrm{L}(\mathrm{EFD})$ over $\Sigma$, and one can see that it is terminating since each rule is size reducing.

We now proceed by performing critical pair analysis on $T$. There are ten critical pairs, all but one of which are strongly joinable apart from one (Fig. 8). Now observe that Lemma 2 tells us that EFDs cannot contain such cycles. With this knowledge, we define $\mathcal{D}$ to be all graphs such that directed cycles contain at least one $t$-labelled edge. Clearly, $\mathcal{D}$ is subgraph closed, and then by our Generalised Critical Pair Lemma (Theorem 9), we have that $T$ is locally confluent on $\mathcal{D}$.

Next, it is easy to see that $\mathcal{D}$ is closed under $T$, so we can use Generalised Newman's Lemma (Theorem 8) to conclude confluence on $\mathcal{D}$ and thus, by Proposition $1, T$ is confluent on $\mathrm{L}(\mathrm{EFD})$.

Thus, $T$ is a confluent decider for $\mathrm{L}(\mathrm{EFD})$ over $\Sigma$, as required.
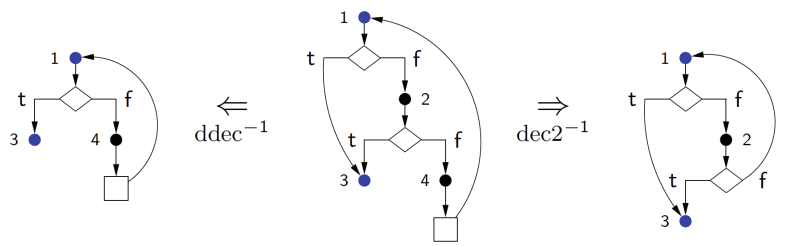

Fig. 8. Non-joinable EFD critical pair 


\subsection{Series-Parallel Graphs}

Series-parallel graphs were introduced by Duffin [9] as a model of electrical networks. A more general version of the class was introduced by Lawler [23] and Monma and Sidney [24] as a model for scheduling problems.

Definition 9. Series-parallel graphs are inductively defined:

1. $P$ is a series-parallel graph where $s$ is the source and $t$ the sink.

2. The class of series-parallel graphs is closed under parallel composition and sequential composition.

where $P=: \rightarrow_{\mathrm{t}}$, parallel composition identifies the two sources and the two sinks, and sequential composition identifies the sink of one with the source of another.

Duffin showed that a graph is series-parallel if and only if it can be reduced to $P$ by a sequence of series and parallel reductions. We can rephrase this in terms of a graph grammar.

Theorem 13 (SP Recognition [29]). The class of series-parallel graphs is the language generated by grammar $S P=((\{\square\},\{\square\}),(\emptyset, \emptyset),\{s, p\}, P)$ (Fig. 9).

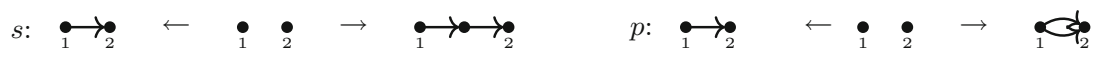

Fig. 9. Series-parallel grammar rules

By traditional critical pair analysis, one can establish that the reversed rules are confluent, however, we run into a problem if we want to consider arbitrarily labelled graphs. Consider the case where the edge alphabet is of size 2, rather than size 1 . The obvious modification to the rules is to use all combinations of labels in LHS graphs (Fig. 10), however Hristakiev and Plump [16] observed that when doing (the equivalent of) this in GP 2, we no longer have confluence. We exhibit a counter example to confluence in Fig. 11.

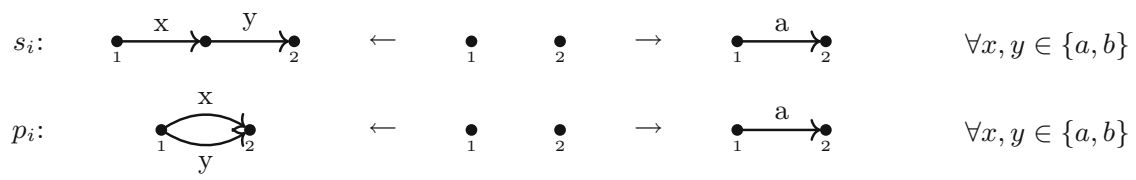

Fig. 10. Labelled series-parallel reduction rules

All is not lost, however, because we can use our new theory to show that, via non-garbage critical pair analysis, the new system is confluence up to garbage, and so we can show that we have a confluent decider. Moreover, our system for two edge labels can be easily generalised for any finite edge alphabet. 


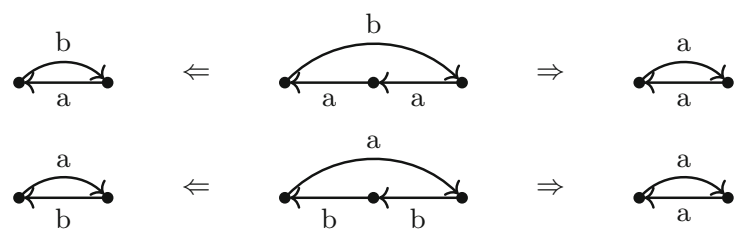

Fig. 11. Non-joinable labelled series-parallel pairs

Definition 10. The class of labelled series-parallel graphs (LSPs) is all seriesparallel graphs, but with arbitrary edge labels chosen from $\Sigma_{E}=\{a, b\}$.

Theorem 14 (Confluent LSP Decider). Let $\Sigma=(\{\square\},\{a, b\}), T=(\Sigma$,

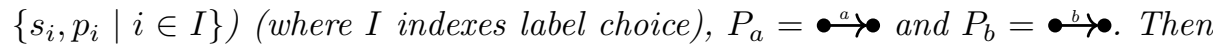
$\left(T,\left\{P_{a}, P_{b}\right\}\right)$ is a confluent decider for the labelled series-parallel graphs over $\Sigma$.

Proof. We denote by $\mathcal{L}$ the language of all labelled series-parallel graphs.

Our rules are structurally the same as the unlabelled rules, so because our LHS graphs are arbitrarily labelled, language recognition of $\mathcal{L}$ over $\Sigma$ follows from Theorem 13. Termination follows from the fact that the combined metric of graph size plus number of $b$-labelled edges strictly decreases with each derivation.

We now proceed by performing critical pair analysis on $T$. We find that we have two non-isomorphic critical pairs that are not joinable (Fig. 11). These pairs have a cyclic start graph, but the series-parallel graphs are acyclic, so we can define $\mathcal{D}$ to be the language of acyclic graphs over $\Sigma$, thus classifying these two pairs as garbage. The remaining critical pairs are strongly joinable, so by Theorem 9, we have that $T$ is locally confluent on $\mathcal{D}$.

We find that all the non-garbage critical pairs are strongly joinable. We have, up to isomorphism, two garbage critical pairs. These are not even joinable, which give us a counter example to (local) confluence, but since all our non-garbage pairs are strongly joinable, we can claim local confluence up to garbage.

Next, it is easy to see that $\mathcal{D}$ is closed under $T$, so we can use Theorem 8 to conclude confluence on $\mathcal{D}$ and thus, by Proposition 1, $T$ is confluent on $\mathcal{L}$.

\section{Conclusion and Future Work}

In this paper we have introduced (local) confluence and termination up to garbage for DPO graph transformation systems, and shown that Newmann's Lemma and Plump's Critical Pair Lemma can be generalised, providing us with machine checkable conditions for confluence up to garbage, using only critical pairs. Of course, confluence up to garbage of terminating graph transformation systems is undecidable in general, however, now we can detect more positive cases of confluence up to garbage using non-garbage critical pair analysis, where we previously would have been unable to draw a conclusion due to non-strong joinability of some critical pairs. 
In particular, our results can be directly applied to recognition of languages, which we have demonstrated with extended flow diagrams and labelled seriesparallel graphs. We have backtracking-free algorithms that apply reduction rules as long as possible, with correctness established via non-garbage critical pair analysis. We also anticipate there to be other applications, since there are many other reasons one would want to show confluence up to garbage, such as considering GT systems as computing functions where we restrict [15]. Indeed, one might only be interested in the non-garbage critical pairs themselves, and classification of conflicts $[20,22]$.

Confluence analysis of GT systems (and related systems) still remains a generally under-explored area. One obvious piece of future work is to investigate the connection to the work by Lambers, Ehrig and Orejas on essential critical pairs [21] and the continued work by others including Born and Taentzer [20]. It is also not obvious if there is a relation between confluence up to garbage and graphs satisfying negative constraints [19]. Moreover, developing a stronger version of the Generalised Critical Pair Lemma that allows for the detection of persistent nodes that need not be identified in the joined graph would allow conclusions of confluence up to garbage where it was previously not determined.

Future work also includes developing checkable sufficient conditions under which one can decide if a graph is in the subgraph closure of a language. Finally, applying our theory in a rooted context and to GP 2 is future work [2]. It is likely that the theory will be applicable there, since program preconditions correspond exactly to non-garbage input, and so it is only natural to be interested in confluence up to garbage, rather than confluence. We would also expect there to be analogues of our results for other kinds of rewriting systems such as string and term rewriting.

\section{References}

1. Baader, F., Nipkow, T.: Term Rewriting and All That. Cambridge University Press, Cambridge (1998)

2. Bak, C.: GP 2: efficient implementation of a graph programming language. Ph.D. thesis, Department of Computer Science, University of York, UK (2015). https:// etheses.whiterose.ac.uk/12586/

3. Bakewell, A., Plump, D., Runciman, C.: Specifying pointer structures by graph reduction. In: Pfaltz, J.L., Nagl, M., Böhlen, B. (eds.) AGTIVE 2003. LNCS, vol. 3062, pp. 30-44. Springer, Heidelberg (2004). https://doi.org/10.1007/978-3-54025959-6_3

4. Berstel, J.: Transductions and Context-Free Languages. Vieweg+Teubner, Stuttgart (1979). https://doi.org/10.1007/978-3-663-09367-1

5. Campbell, G.: Efficient graph rewriting. BSc thesis, Department of Computer Science, University of York, UK (2019). https://arxiv.org/abs/1906.05170

6. Campbell, G., Plump, D.: Efficient recognition of graph languages. Technical report, Department of Computer Science, University of York, UK (2019). https:// arxiv.org/abs/1911.12884

7. Caron, A.-C.: Linear bounded automata and rewrite systems: influence of initial configurations on decision properties. In: Abramsky, S., Maibaum, T.S.E. (eds.) 
CAAP 1991. LNCS, vol. 493, pp. 74-89. Springer, Heidelberg (1991). https://doi. org/10.1007/3-540-53982-4_5

8. Courcelle, B.: The monadic second-order logic of graphs: definable sets of finite graphs. In: van Leeuwen, J. (ed.) WG 1988. LNCS, vol. 344, pp. 30-53. Springer, Heidelberg (1989). https://doi.org/10.1007/3-540-50728-0_34

9. Duffin, R.J.: Topology of series-parallel networks. J. Math. Anal. Appl. 10(2), 303-318 (1965). https://doi.org/10.1016/0022-247X(65)90125-3

10. Ehrig, H., Ehrig, K., Prange, U., Taentzer, G.: Fundamentals of Algebraic Graph Transformation. Monographs in Theoretical Computer Science. An EATCS Series. Springer, Heidelberg (2006). https://doi.org/10.1007/3-540-31188-2

11. Ehrig, H., Kreowski, H.-J.: Parallelism of manipulations in multidimensional information structures. In: Mazurkiewicz, A. (ed.) MFCS 1976. LNCS, vol. 45, pp. 284-293. Springer, Heidelberg (1976). https://doi.org/10.1007/3-540-07854-1_188

12. Ehrig, H., Pfender, M., Schneider, H.: Graph-grammars: an algebraic approach. In: Proceedings 14th Annual Symposium on Switching and Automata Theory, SWAT 1973, pp. 167-180. IEEE (1973). https://doi.org/10.1109/SWAT.1973.11

13. Farrow, R., Kennedy, K., Zucconi, L.: Graph grammars and global program data flow analysis. In: Proceedings 17th Annual Symposium on Foundations of Computer Science, SFCS 1976, pp. 42-56. IEEE (1976). https://doi.org/10.1109/SFCS. 1976.17

14. Fradet, P., Métayer, D.L.: Structured gamma. Sci. Comput. Program 31(2-3), 263-289 (1998). https://doi.org/10.1016/S0167-6423(97)00023-3

15. Habel, A., Müller, J., Plump, D.: Double-pushout graph transformation revisited. Math. Struct. Comput. Sci. 11(5), 637-688 (2001). https://doi.org/10.1017/ S0960129501003425

16. Hristakiev, I., Plump, D.: Checking graph programs for confluence. In: Seidl, M., Zschaler, S. (eds.) STAF 2017. LNCS, vol. 10748, pp. 92-108. Springer, Cham (2018). https://doi.org/10.1007/978-3-319-74730-9_8

17. Huet, G.: Confluent reductions: abstract properties and applications to term rewriting systems. J. ACM 27(4), 797-821 (1980). https://doi.org/10.1145/322217. 322230

18. Knuth, D., Bendix, P.: Simple word problems in universal algebras. In: Leech, J. (ed.) Computational Problems in Abstract Algebra, pp. 263-297. Pergamon Press, Oxford (1970). https://doi.org/10.1016/B978-0-08-012975-4.50028-X

19. Lambers, L.: Certifying rule-based models using graph transformation. Ph.D. thesis, Technical University of Berlin, Elektrotechnik und Informatik (2009). https:// doi.org/10.14279/depositonce-2348

20. Lambers, L., Born, K., Orejas, F., Strüber, D., Taentzer, G.: Initial conflicts and dependencies: critical pairs revisited. In: Heckel, R., Taentzer, G. (eds.) Graph Transformation, Specifications, and Nets. LNCS, vol. 10800, pp. 105-123. Springer, Cham (2018). https://doi.org/10.1007/978-3-319-75396-6_6

21. Lambers, L., Ehrig, H., Orejas, F.: Efficient conflict detection in graph transformation systems by essential critical pairs. In: Proceedings Fifth International Workshop on Graph Transformation and Visual Modeling Techniques, GT-VMT 2006, Electronic Notes in Theoretical Computer Science, vol. 211, pp. 17-26. Elsevier (2008). https://doi.org/10.1016/j.entcs.2008.04.026

22. Lambers, L., Kosiol, J., Strüber, D., Taentzer, G.: Exploring conflict reasons for graph transformation systems. In: Guerra, E., Orejas, F. (eds.) ICGT 2019. LNCS, vol. 11629, pp. 75-92. Springer, Cham (2019). https://doi.org/10.1007/978-3-03023611-3_5 
23. Lawler, E.: Sequencing jobs to minimize total weighted completion time subject to precedence constraints. Ann. Discrete Math. 2, 75-90 (1978). https://doi.org/10. 1016/S0167-5060(08)70323-6

24. Monma, C., Sidney, J.: Sequencing with series-parallel precedence constraints. Math. Oper. Res. 4(3), 215-224 (1979). https://doi.org/10.1287/moor.4.3.215

25. Newman, M.: On theories with a combinatorial definition of "equivalence". Ann. Math. 43(2), 223-243 (1942). https://doi.org/10.2307/1968867

26. Plump, D.: Hypergraph rewriting: critical pairs and undecidability of confluence. In: Sleep, M.R., Plasmeijer, M.J., van Eekelen, M.C. (eds.) Term Graph Rewriting, pp. 201-213. Wiley, Chichester (1993)

27. Plump, D.: Computing by graph rewriting. Habilitation thesis, Universität Bremen, Fachbereich Mathematik und Informatik (1999)

28. Plump, D.: Confluence of graph transformation revisited. In: Middeldorp, A., van Oostrom, V., van Raamsdonk, F., de Vrijer, R. (eds.) Processes, Terms and Cycles: Steps on the Road to Infinity. LNCS, vol. 3838, pp. 280-308. Springer, Heidelberg (2005). https://doi.org/10.1007/11601548_16

29. Plump, D.: Reasoning about graph programs. In: Proceedings 9th International Workshop on Computing with Terms and Graphs, TERMGRAPH 2016, Electronic Proceedings in Theoretical Computer Science, vol. 225, pp. 35-44. Open Publishing Association (2016). https://doi.org/10.4204/EPTCS.225.6 